\title{
Role of lacrimal scintigraphy and subtraction macrodacryocystography in the management of epiphora
}

\author{
L. A. AMANAT, ${ }^{1}$ E. P. WRAIGHT, ${ }^{2}$ P. G. WATSON, ${ }^{1}$ and T. D. HAWKINS ${ }^{3}$ \\ From the Departments of ${ }^{1}$ Ophthalmology, ${ }^{2}$ Nuclear Medicine, and ${ }^{3}$ Diagnostic Radiology, \\ Addenbrooke's Hospital, Cambridge
}

SUMMARY Fifty-one patients were investigated by subtraction macrodacryocystography (SMDCG, 103 systems) and by lacrimal scintigraphy (LS, 105 systems). It was found that these investigations complemented each other and between them the precise site of obstruction in the lacrimal drainage apparatus could be determined in $80 \%$. The radiation dosage to the lens in SMDCG is significant, and it is therefore recommended that the patients with lacrimal obstruction should: (1) have lacrimal puncta dilated with a probe to No. 1 diameter and be forcibly syringed; (2) have lacrimal scintigraphy performed; (3) if the site of the obstruction is still uncertain, then and only then should SMDCG be performed.

Epiphora can often be managed by listening to the history, examining the lids, and syringing the lacrimal drainage apparatus. However, this will not always determine the site and nature of the obstruction, and further investigations can then be of assistance.

Dacryocystography has been performed since 1909 (Ewing, 1909). The technique was much improved by Lloyd (1973), who used intubation and subtraction macrodacryocystography (SMDCG). This technique enables the lacrimal system to be outlined in detail, but the results are sometimes misleading, particularly in common canalicular obstruction and in patients with a functional block (Demorest and Milder, 1955), that is, patients with epiphora and normal classical dacryocystography.

Rossomondo et al. (1972) demonstrated the passage of $99 \mathrm{~m}$ technetium through the lacrimal drainage apparatus with a gamma camera and a specially developed micropinhole collimator (Microscintigraphy, Lacrimal Scintigraphy, Lacrimal Scintillography). By this technique the dynamics of the lacrimal drainage apparatus could be studied under much more physiological conditions. Hurwitz et al. (1975a) and Chaudhari et al. $(1974,1975)$ have used both the subtraction macrodacryocystogram and lacrimal scintigraphy to assess the lacrimal drainage

Address for reprints: Dr L. A. Amanat, Tennent Institute of Ophthalmology, Western Infirmary, Glasgow G11 6NT in patients complaining of epiphora, and arrived at different conclusions.

The purpose of this paper is to assess again the value of these techniques in ordinary hospital practice and to compare them with simple clinical observations in order to determine whether either method should be used to decide the operative procedure. In addition, the effects of those treatments have been evaluated.

\section{Material and methods}

All patients who attended the Eye Department of Addenbrooke's Hospital, Cambridge, and Huntingdon County Hospital, Huntingdon, complaining of epiphora over a period of 2 years were included in the study.

All patients had a careful examination to detect any obvious lid abnormalities such as ectropion, eversion of punctum, or entropion. This was followed by syringing of the lacrimal drainage apparatus, subtraction macrodacryocystography (SMDCG), and lacrimal scintigraphy (LS).

The nasolacrimal drainage system was studied bilaterally in 51 patients, except in 1 patient who was 1 -eyed and therefore 1 side only was studied. Ten of these were male patients (this included a 3-yearold boy) and 41 were female patients. Two patients had both the SMDCG and LS repeated, 1 patient had the LS only repeated, while 2 patients did not 
Table 1 Result of dacryocystorhinostomy

\begin{tabular}{|c|c|c|c|c|}
\hline \multirow{2}{*}{ Patient no. } & \multicolumn{2}{|l|}{$S M D C G$ result } & \multicolumn{2}{|l|}{$L S$ result } \\
\hline & Preoperative & Postoperative & Preoperative & Postoperative \\
\hline 4 (R) & NL duct obstruction & DCR patent & Canalicular obstruction & Lacrimal sac obstruction \\
\hline 11 (L) & No obstruction & Free flow & $\begin{array}{l}\text { Lacrimal sac obstruction } \\
\quad \text { (partial) }\end{array}$ & Lacrimal sac obstruction \\
\hline 47 (L) & NL duct obstruction & Free flow & NL duct obstruction & Not done \\
\hline 54 (L) & Canalicular obstruction & Canalicular obstruction & Canalicular obstruction & Canalicular obstruction \\
\hline
\end{tabular}

All patients were asymptomatic except Patient 54. NL = nasolacrimal

Table 2 Results of dacryocystorhinostomy with silicone tubes

\begin{tabular}{|c|c|c|c|c|c|}
\hline \multirow{2}{*}{\multicolumn{2}{|c|}{ Patient no. }} & \multicolumn{2}{|l|}{$S M D C G$ result } & \multicolumn{2}{|l|}{$L S$ result } \\
\hline & & \multirow{2}{*}{$\begin{array}{l}\text { Preoperative } \\
\text { Upper and lower } \\
\text { canalicular obstruction }\end{array}$} & \multirow{2}{*}{$\begin{array}{l}\text { Postoperative } \\
\text { Free flow }\end{array}$} & \multirow{2}{*}{$\begin{array}{l}\text { Preoperative } \\
\text { Canalicular obstruction }\end{array}$} & \multirow{2}{*}{$\begin{array}{l}\text { Postoperative } \\
\text { Improved flow }\end{array}$} \\
\hline 1 & (R) & & & & \\
\hline 3 & (L) & Canalicular obstruction & Canalicular obstruction & Canalicular obstruction & Canalicular obstruction \\
\hline 7 & ( $R$ and $L$ ) & Canalicular obstruction & Free flow & Canalicular obstruction & Good flow \\
\hline 13 & ( $R$ and $L)$ & Not done & Canalicular obstruction & Canalicular obstruction & Canalicular obstruction \\
\hline 20 & (R) & $\begin{array}{l}\text { Upper and lower } \\
\text { canalicular obstruction }\end{array}$ & Not done & Canalicular obstruction & Free flow \\
\hline 29 & (R) & $\begin{array}{l}\text { NL duct obstruction } \\
\text { (partial) }\end{array}$ & Slow but definite flow & NL duct obstruction & Slow but definite flow \\
\hline 35 & (R) & Canalicular obstruction & Lacrimal sac obstruction & Canalicular obstruction & Lacrimal sac obstruction \\
\hline 43 & (L) & Canalicular obstruction & Free flow & Canalicular obstruction & Free flow \\
\hline 57 & (L) & Canalicular obstruction & DCR patent & $\begin{array}{l}\text { Canalicular obstruction } \\
\text { (partial) }\end{array}$ & $\begin{array}{l}\text { Canalicular obstruction } \\
\text { (partial) }\end{array}$ \\
\hline
\end{tabular}

All patients were asymptomatic except Patient 13

have an SMDCG. Thus, in all, 103 systems were studied by SMDCG and 105 systems were studied by LS. Most patients who required some form of surgery had SMDCG and LS repeated after the operation, the time interval varying from patient to patient depending on the nature of surgery. The preoperative findings in patients who required a dacryocystorhinostomy (DCR) or a DCR with silicone tubes are compared with those obtained postoperatively, in Tables 1 and 2 respectively. It is of interest to note that although the symptoms were relieved in all but 1 patient, who had dacryocystorhinostomy with silicone tubes, obstruction in the lacrimal drainage system could be demonstrated postoperatively by SMDCG and LS in 2 other systems (Patients 3 and 35). In patients who had dacryocystorhinostomy alone without silicone tubes LS remained abnormal in those in whom it was possible to carry out this investigation. In addition
6 of the 14 patients who improved symptomatically after the investigations had the SMDCG and LS repeated to ascertain any difference in the results of the investigations (Table 3). In 4 patients the obstruction was no longer demonstrable on SMDCG and/or LS.

TECHNIQUE OF LACRIMAL SCINTIGRAPHY After the procedure has been explained the patient sits erect on a chair in front of the $\frac{3}{16}$-in $(4 \cdot 5-\mathrm{mm})$ pinhole collimator, with the chin and forehead stabilised on a stand similar to the one attached to a slit lamp. A band is secured around the patient's head to minimise involuntary movements. The distance between the collimator and cornea is approximately $50 \mathrm{~mm}$.

Immediately before the radioactive isotope is instilled the lacrimal sacs are emptied by digital expression and immediately afterwards $15 \mu \mathrm{l}$ con- 
Table 3 Comparison of results in patients who became asymptomatic

\begin{tabular}{llllll}
\hline Patient no. & \multicolumn{2}{l}{ SMDCG result } & & LS result \\
\cline { 3 - 5 } & & First visit & Second visit & First visit & Second visit \\
\hline 5 & (R) & NL duct obstruction & No obstruction & No obstruction & ? Partial hold-up at NL duct \\
9 & (L) & Lacrimal sac obstruction & Lacrimal sac obstruction & Canalicular obstruction & Unsatisfactory \\
17 & (L) & NL duct obstruction & No obstruction & NL duct obstruction & Normal \\
37 & (L) & Lacrimal sac obstruction & No obstruction & Canalicular obstruction & Lacrimal sac obstruction \\
50 & (R) & Difficult to cannulate & No obstruction & Normal & Normal \\
& (L) & No obstruction & No obstruction & Canalicular obstruction & Canalicular obstruction \\
51 & (R) & Normal & No obstruction & Normal & Normal \\
& (L) & Normal & No obstruction & Canalicular obstruction & Normal \\
\end{tabular}

taining about $50 \mu \mathrm{Ci}$ of $99 \mathrm{~m}$ technetium sulphur colloid is delivered simultaneously to the conjunctival sacs at the lateral canthus by means of automatic Finnpipettes. Gama camera data acquisition is started at the same moment. The passage of the tracer is displayed on an oscilloscope, and serial images are recorded at 20-second intervals for 10 minutes on the magnetic disc of a computer system (Varicom-Varian 620L). The data on the magnetic

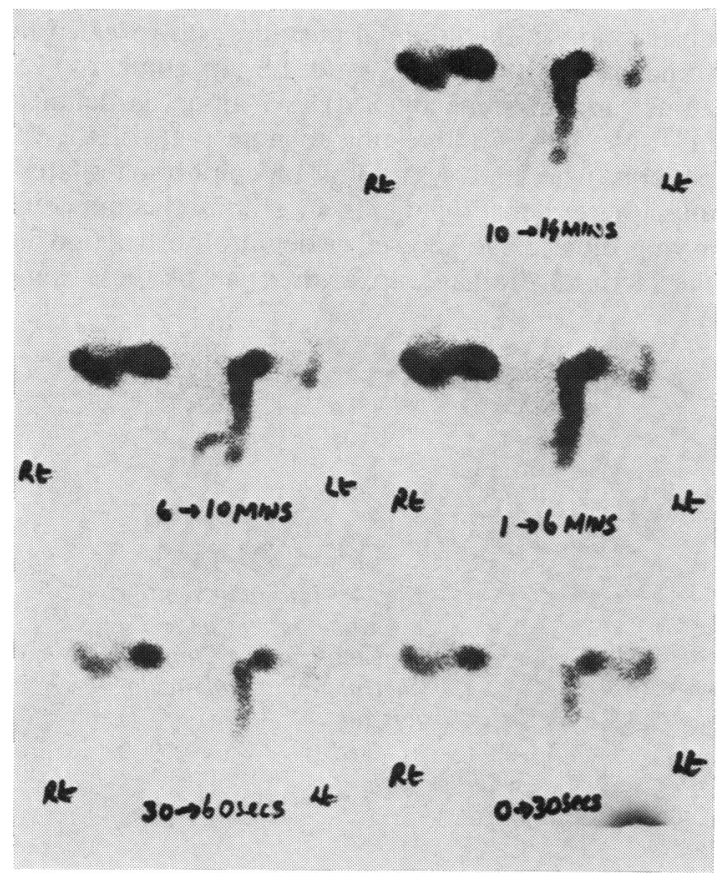

Fig. 1 Lacrimal scintigram of a patient showing obstruction at canalicular level on right side disc are later transferred to a tape. After the study has been completed the data are analysed.

Static views are reproduced on a hard copy showing the pattern of lacrimal flow at 1 to 20 seconds, 21 to 40 seconds, 1 to 2 minutes, 3 to 4 minutes, 5 to 6 minutes, 7 to 8 minutes, 9 to 10 minutes, and a final copy showing the 'sum' view from 0 to 10 minutes.

A suitable view is then displayed on the oscillo-

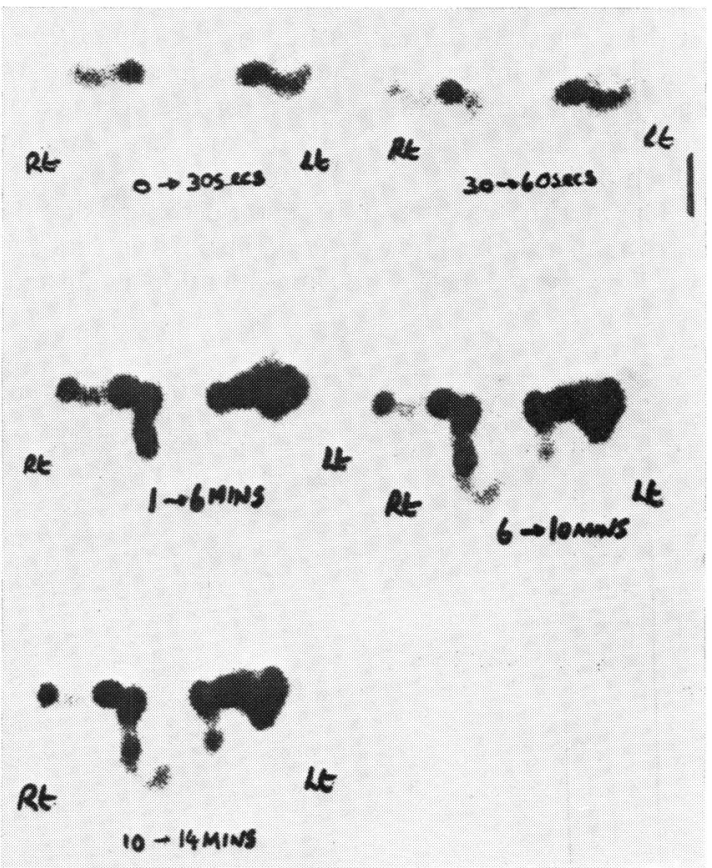

Fig. 2 Lacrimal scintigram of a patient showing obstruction at lower end of lacrimal sac on left side 


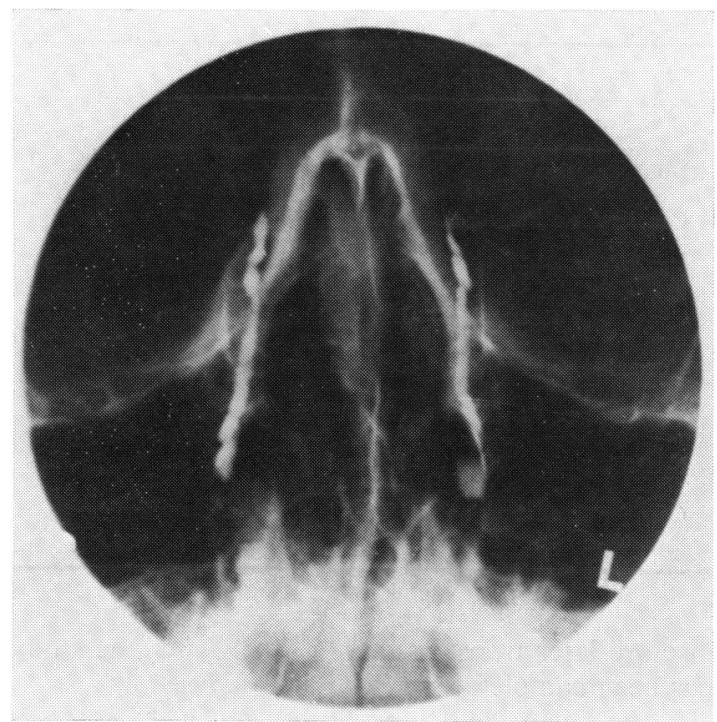

Fig. 3 Normal macrodacryocystogram of a patient complaining of persistent left epiphora

scope and 5 areas of interest are defined on each side outlining the conjunctival sac, medial canthus, lacrimal sac, nasolacrimal duct, and the nasal cavity.

Dynamic curves of changes in activity with time in these regions are then plotted by the computer.

The serial images are interpreted in the light of the dynamic curves and assessed for the presence of complete or partial obstruction and the level at which it occurs.

The lacrimal scintigraphy method differs slightly from that used by Hurwitz et al. (1975b) in that the sac is expressed before the investigation is started, thus enabling the lacrimal sac to be more readily visualised by emptying it from any accumulated tears.

\section{TECHNIQUE OF SUBTRACTION MACRODACR YOCYSTOGRAPHY}

The macrodacryocystography technique used for the examination of the patient in this study was that described by Lloyd (1973). The patient is examined in the supine position with the orbits at a level approximately two-thirds the distance between the tube target and the film to produce an enlarged image of about $\times 1.5$ magnification. The $x$-ray tube has a $0.3-\mathrm{mm}$ focal spot to reduce geometric blur. The lower or occasionally the upper canaliculus is catheterised with a fine nylon tube, and a film for subtraction is taken immediately before the injection of contrast medium. A series of 3 or 4 films are taken during the injection of 1.0 to $1.5 \mathrm{ml}$ of iodised oil (Lipiodol ultra fluid). A positive image of the film taken for subtraction purposes is superimposed on one or more of the films taken during the injection of contrast medium to obtain a bone-free image of the contrast-filled duct system.

\section{Results and analysis}

The total number of patients studied was 51 . The obstruction in the lacrimal passages was revealed in some by both SMDCG and LS, in some by LS alone, and in some by SMDCG alone as detailed in Table 4. It is interesting to note that, of the 25 patients who had symptoms of epiphora unilaterally, there were 7 patients in whom the opposite asymptomatic eye showed a definite obstruction in the lacrimal drainage apparatus; six of these were

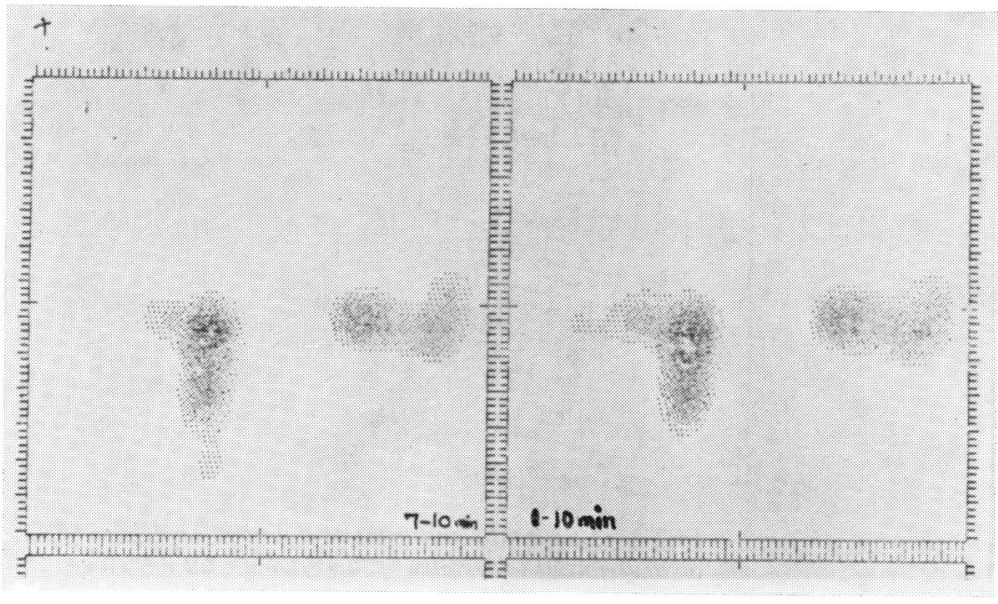

Fig. 4 Lacrimal scintigram of the same patient as in Fig. 3 showing complete functional obstruction at canalicular level on left side 


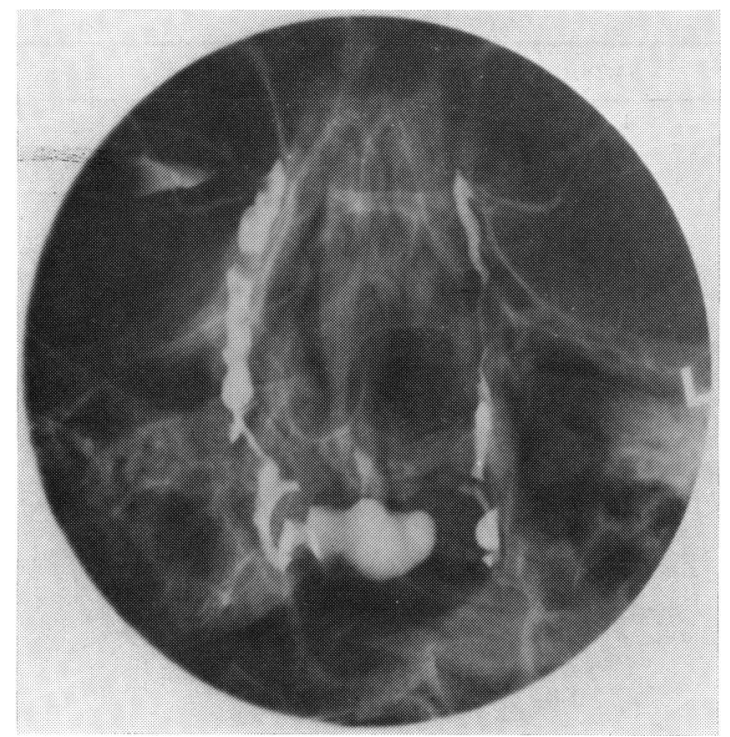

Fig. 5 Macrodacryocystogram showing stenosis and partial obstruction of right nasolacrimal duct with consequent dilatation of lacrimal sac

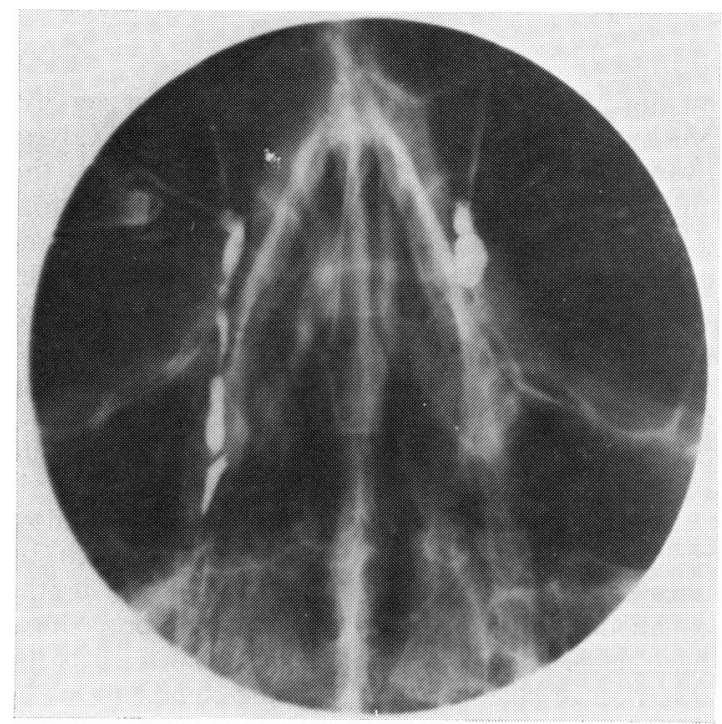

Fig. 6 Macrodacryocystogram showing obstruction at the lower end of lacrimal sac on left side

demonstrated by LS alone and one by both SMDCG and LS.

The exact sites of obstruction, as determined by syringing and SMDCG and LS, are compared in Table 5 . It is evident from Table 5 that a definite level of obstruction could be determined by syringing in 34 out of 77 systems ( $44 \%$ ), by SMDCG in 44 out of 79 systems $(55.7 \%)$, and by LS in 62 out of 81 systems $(76 \cdot 6 \%)$. It should be noted that stasis due to distal obstruction may result in hold-up of tracer more proximally and lead to error in determining the site of obstruction by LS.

Twenty-six patients complained of bilateral epiphora, and 25 patients complained of unilateral epiphora; altogether 81 symptomatic systems were studied in these patients. In 41 systems the obstruction was picked up by both the SMDCG and LS (Table 4, excluding one asymptomatic system); SMDCG alone revealed an obstruction in 3 systems; and LS alone revealed an obstruction in 21 eyes (Table 6 , excluding the 6 asymptomatic systems). Thus between them SMDCG and LS were able to demonstrate an obstruction in 65 out of the 81 symptomatic systems $(80 \%)$.

\section{Discussion}

Epiphora is never dangerous and at worst causes excoriation of the skin, but is nonetheless an extremely irritating symptom, made worse by exposure to wind and sun, often preventing the patient from working properly because of the constant streaming

Table 4 Method by which obstruction was revealed

(A) Symptoms of bilateral epiphora present in

26 Patierts (56 systems)

(i) Bilateral obstruction demonstrated in 14 patients ( 30 systems)* By both SMDCG and LS in 17 systems By LS alone in 13 systems

(ii) Unilateral obstruction demonstrated in 10 patients ( 10 systems) By both SMDCG and LS in 6 systems By LS alone in 3 systems By SMDCG alone in

1 system

(iii) No obstruction could be demonstrated in 3 patients (6 systems) $\dagger$

(B) Symptoms of unilateral epiphora present in

25 Patients (50 syste'ns)

(i) Unilateral obstruction demonstrated in 18 patients (18 systems) By both SMDCG and LS in 13 systems By LS alone in 3 systems By SMDCG alone in

2 systems

(ii) Bilateral obstruction demonstrated in 7 patients (14 systems) Symptomatic

By both SMDCG and LS in 5 systems By LS alone in 2 systems

Asymptomatic

By both SMDCG and LS in 1 system

By LS alone in 6 systems

*One patient had the SMDCG and the LS repeated so that he had 4 systems investigated. TOne of these patients had the investigations done twice; on the first occasion she showed a unilateral obstruction only and so this patient is also included in subgroup (ii). In brief: SMDCG and LS together revealed an obstruction in 42 systems,

LS alone revealed an obstruction in 27 systems, and SMDCG alone revealed an obstruction in 3 systems 
Table 5 Comparison of site of obstruction

\begin{tabular}{lccc}
\hline Level of obstruction & Syringing & SMDCG & LS \\
\hline Upper canaliculus & - & 1 & - \\
Lower canaliculus & 4 & - & - \\
Combined upper and lower canaliculus & - & 2 & 27 \\
Complete obstruction of common canaliculus & 16 & 2 & 4 \\
Partial obstruction of common canaliculus & - & 7 & 12 \\
Complete obstruction of lower end of lacrimal sac & 3 & 1 & 4 \\
Partial obstruction of lower end of lacrimal sac & - & 10 & 5 \\
Complete obstruction of nasolacrimal duct & 11 & 6 & 4 \\
Partial obstruction of nasolacrimal duct & - & - & 6 \\
Level of obstruction difficult to ascertain & 20 & 35 & 19 \\
No obstruction demonstrated in symptomatic eyes & 18 & - & - \\
\hline Level of obstruction not recorded & 5 & 79 & 81 \\
\hline Asymptomatic systems* & 77 & 24 & 24 \\
\hline Total systems & -77 & 103 & 105 \\
\hline
\end{tabular}

Syringing revealed an obstruction in 34 out of 77 systems (44\%). SMDCG revealed an obstruction in 44 out of 79 systems (55.7\%). LS revealed an obstruction in 62 out of 81 systems $(76.6 \%)$. * Seven of these showed an obstruction in the lacrimal drainage apparatus

of tears on to the face. It is always a great social embarrassment. The patient therefore demands relief, which can usually be effected provided the appropriate treatment is given. This depends on determining accurately the site of obstruction to the outflow of tears from the eye. The tears will overflow from the eye if the lids are lax and allow the punctum to be everted, if either the upper or the lower punctum is everted, if either the upper or the lower punctum is stenosed, if the lumen of the canaliculi are occluded or they are constricted from without

Table 6 Level of obstruction on LS when SMDCG was normal

\begin{tabular}{lc}
\hline Complete canlicular obstruction & 5 systems \\
Partial canalicular obstruction & 1 system \\
Complete obstruction at the lower end of lacrimal sac & 10 systems \\
Partial obstruction at the lower end of lacrimal sac & 3 systems \\
Complete obstruction at nasolacrimal duct & 3 systems \\
Partial obstruction at nasolacrimal duct & 2 systems \\
Total & 24 systems \\
\hline
\end{tabular}

In addition to these 24 systems there were 3 systems which also revealed an obstruction on LS, but as a SMDC (3 was not done on these they are not included in the Table. The Table includes the 6 systems which were asymptomatic contralateral eyes by fibrosis or aberrant muscle action, or by the presence of abnormal mucous membrane valves. Even if the tears do enter the lacrimal sac, the outflow from the sac may be obstructed by malformations, infection or damage to the mucous membrane valves of the sac itself. As the abnormalities of the lids and puncta can be dealt with easily by eyelid tightening procedures, retropunctal cautery, dilatation of the puncta, or the permanent opening of the puncta by the three-snip procedure, it is most important to establish that this is the sole site of faulty lacrimal drainage.

Lesions of the lacrimal sac and the outflow mechanisms of the sac are easily dealt with by dacryocystorhinostomy (DCR). However, if the canaliculi are obstructed, more complex procedures have to be considered, the simplest of which is the insertion of silicone tubes into the canaliculi at the time a DCR is performed.

In recent years subtraction macrodacryocystography and lacrimal scintigraphy have been added to the armamentarium of procedures available for the investigation of epiphora. It has been argued that they are unnecessary and that simply syringing the tear ducts will give all the information that is required. This is not borne out by this study. In fact syringing revealed an obstruction in only $44 \%$ of those who complained of symptoms, whereas 
Table 7 Follow-up of patients in whom SMDCG was normal

22 systems were contralateral asymptomatic systems
(these include 6 systems in whom LS showed a definite
obstruction)
5 systems improved after investigations
16 systems required no treatment as symptoms were not very
severe
2 systems are being treated conservatively
9 systems required surgery*
2 systems awaiting a decision as regards treatment
1 system refused treatment

Total 57 systems

* One had DCR, 2 had DCR with tubes, 3 had three-snip operations, 2 had probing, 1 had retropunctal cautery. Three of these patients also showed normal LS, and are included in Table 10 as well

SMDCG and LS used together showed a definite obstruction in $80 \%$ of patients.

Lacrimal scintigraphy succeeded in revealing an obstruction in $76.6 \%$ of the symptomatic systems investigated, and the exact level of the obstruction could be determined in all but 6 systems $(10 \%)$ (Table 5). Of the remaining 19 symptomatic eyes, which showed no obstruction, 3 improved symptomatically after the investigations; 10 did not require any treatment, as symptoms were not severe; 1 patient was treated conservatively with zinc sulphate drops; and only 5 systems required surgery (4 threesnips, 1 DCR with tubes); and even in this latter patient there was a suggestion that the obstruction was at the lower punctum because there was difficulty in trying to cannulate the inferior punctum when SMDCG was performed. It can therefore be said that in this series lacrimal scintigraphy not only showed an obtruction to be present in the majority of patients, but also showed its exact site and revealed those patients in whom either no treatment was going to be necessary or only simple surgery would succeed.

Subtraction macrodacryostography demonstrates the lacrimal system well but is unphysiological in that the contrast medium can be forced through under pressure when it would not pass under normal circumstances. Some anomaly was shown in the lacrimal passages in $55.7 \%$ of those investigated with SMDCG; the exact site could be determined in all of them (Table 5). Of those in whom SMDCG failed to show an obstruction, 5 systems $(9 \%)$ improved spontaneously, presumably owing to stretching of the canaliculi during the procedure, and a further $16(28 \%)$ were improved to the extent that no further treatment is contemplated
(Table 7). Nine patients $(16 \%)$ required surgery (3 of these also showed a normal LS and these have been discussed with LS above); 3 of these required some form of DCR; 2 resolved on further probing; and 1 required the puncta replacing against the globe, while 3 had three-snip operations. In some cases there seems to be a marked therapeutic benefit from actually performing an SMDCG, but this could be achieved by using a wider canula or a No. 1 probe when syringing the ducts initially.

Table 4 shows that rather than being mutually exclusive LS and SMDCG complemented each other. In each of the categories investigated the site of obstruction was revealed by one method and not by the other. One reason for this is possibly because the tracer cannot enter the sac if there is no flow through it; but this cannot be a major factor, because at sites within the lacrimal sac and the nasolacrimal duct, where one would expect the obstruction to show up better with the SMDCG, the number detected by either method was much the same; 24 out of $44(55 \%)$ by SMDCG, and 25 out of $62(40 \%)$ by LS. Using the collimator available to us we were not able to distinguish between upper and lower canalicular block on LS, but these can be easily detected by simple syringing and by SMDCG, as indeed can common canalicular block (Table 2). However, LS seems to show up a higher percentage of canalicular block than SMDCG and syringing, which again could be because the tracer cannot pass into the lacrimal sac through which there is no flow. Again, only rarely does this seem to be the case, because in only 6 patients (Table 8) in whom LS suggested common canalicular obstruction was this found not to be present at operation. In another patient a common canalicular block was suspected, but it was found at operation that the lacrimal sac was completely occupied by a yellow cast. There was therefore no flow at all through the system, and this result confirmed the LS findings of no passage of tracer beyond the medial canthus.

It is interesting to note that the SMDCG revealed both a superior canalicular and an inferior canalicular block on that side. In two other similar situations, where LS showed no passage of tracer beyond the medial canthus, the sac was replaced by small fibrotic masses. In another instance the hold-up of tracer at the medial canthus was due to callous formation at the site of a DCR performed in the past.

It was surprising that the findings by the various methods of investigations did not correspond more often. A definite level of obstruction was revealed in 69 systems, by SMDCG and/or LS (Table 4, excluding the 3 systems which show an obstruction on LS but did not have an SMDCG performed), and yet the level of obstruction as 
Table 8 Comparison of $L S$ and SMDCG results with operative findings

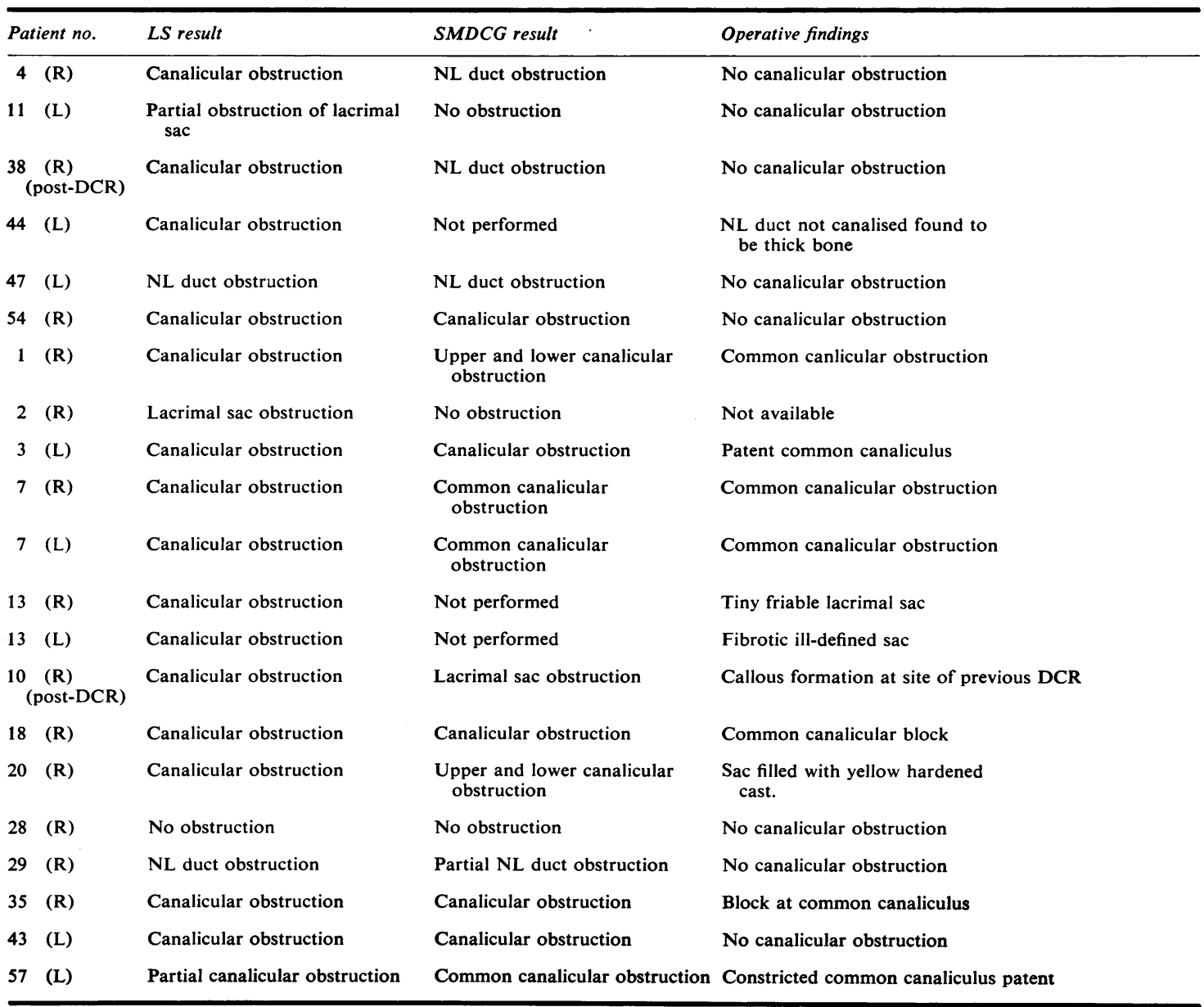

$\mathrm{NL}=$ Nasolacrimal

shown by these two investigations was identical in only 30 systems $(43 \%)$. Eighteen systems showed the obstruction to be present at canalicular level. In 2 of these the obstruction was partial; in 2 the obstruction was at the lower end of the lacrimal sac; while in 6 systems there was complete, and in 2 partial, obstruction at the level of the nasolacrimal duct. If the SMDCG appears to be normal and the scintigram abnormal this is certainly of significance, and records a functional block. A normal SMDCG may be misleading because contrast medium injected through the lacrimal drainage system will force apart mucous membrane surfaces which would otherwise obstruct the flow. LS was performed after the SMDCG in the 3 patients in whom the obstruction was demonstrable on SMDCG only and not on LS, and it is possible that the SMDCG may have relieved the obstruction, thus explaining the absence of detectable obstruction on the LS.

Indeed, it is interesting that $21(41 \%)$ of those investigated did not require any surgery (Table 9). Of those who did, the method of treatment was determined by the results of the investigations. Patients with nasolacrimal duct and lower sac obstruction had a dacryocystorhinostomy performed. Silicone tubes were inserted into the canaliculi in those who had canalicular obstruction; they were also inserted in one patient who had nasolacrimal duct problems, but surgery of the nasal mucous membrane flaps was regarded as inadequate.

In 7 patients 8 systems $(29 \%)$ were treated by simple lid procedures and probing (Table 10). All these patients except one had a normal SMDCG but some abnormality on the LS. This is possibly the 
Table 9 Management of patients after investigations

3 Patients declined surgery

2 Patients were considered unsuitable for surgery*

1 Patient decided to have surgery elsewhere

2 Patients failed to attend regularly-thereafter no treatment

5 Patients being treated conservatively

14 Patients became asymptomatic or epiphora diminished after investigations

24 Patients required some form of surgery

Total 51

- One patient was too ill to undergo surgery; the other patient had had irradiation to inner canthus for basal cell carcinoma on that side in the past

Table 10 Details of lid surgery and probing

\begin{tabular}{|c|c|c|c|}
\hline \multicolumn{2}{|c|}{ Patient no. } & \multirow{2}{*}{$\begin{array}{l}\text { Abnormality on LS } \\
\text { Lacrimal sac obstruction }\end{array}$} & \multirow{2}{*}{$\begin{array}{c}\text { Procedure performed } \\
\begin{array}{c}\text { Three-snip and retro- } \\
\text { punctal cautery }\end{array}\end{array}$} \\
\hline 2 & (R) & & \\
\hline 11 & (R) & Lacrimal sac obstruction & $\begin{array}{l}\text { Probing under general } \\
\text { anaesthetic }\end{array}$ \\
\hline 21 & $\begin{array}{l}\text { (R and } \\
\mathrm{L})\end{array}$ & $\begin{array}{l}\text { Delay in passage of } \\
\text { tracer }\end{array}$ & Bilateral three-snip \\
\hline 22 & (R) & No obstruction & Three-snip \\
\hline 33 & (R) & Canalicular obstruction & $\begin{array}{l}\text { Probing followed by three- } \\
\text { snip }\end{array}$ \\
\hline 40 & (R) & Canalicular obstruction & Retropunctal cautery \\
\hline 53 & (L) & Canalicular obstruction & Retropunctal cautery \\
\hline
\end{tabular}

SMDCG was normal in all these patients except Patient 40, where there was a partial obstruction at the level of the lacrimal sac

result of an abnormality of the lacrimal pump mechanism, because most of these patients show improved flow patterns on lacrimal scintigraphy after surgery and have become asymptomatic. Patient 2, in whom the symptoms persisted, required a DCG with tubes because a repeat LS again showed an obstruction at the level of the lacrimal sac. The symptoms of Patient 53 did not improve after surgery.

The radiation dose to the lens in lacrimal scintigraphy depends to some extent on the degree of retention of tracer in the conjunctival sac. It is usually only a few millirads and does not exceed 20 millirads even in severe obstruction. In contrast the radiation in the area of the lens using the standard techniques of SMDCG, in which the tube distance is very short, is about 2 rads; 200 rads to the lens can produce cataractous changes (Merriam and Focht, 1957). Because of this and of the ability of lacrimal scintigraphy to detect the majority of the obstructions, we would recommend that after syringing the tear duct lacrimal scintigraphy is performed. If doubt still remains as to the site of the obstruction, then and only then should macrodacryocystography be performed.

We thank Mrs S. Smith for her invaluable secretarial assistance and Miss $\mathrm{K}$. Smith for typing the paper.

We also acknowledge the help of the members of the Department of Nuclear Medicine and the Department of Radiology.

\section{References}

Chaudhari, T. K.. Saparoff, G. R., Dolon, K. D., and Chaudhari, T. K. (1974). A comparative study of contrast dacryocystogram and nuclear dacryocystogram. Journal of Nuclear Medicine, 15, 482.

Chaudhari, T. K., Saparoff, G. R., Dolon, K. D., and Chaudhari, T. K. (1975). A comparative study of contrast dacryocystogram and nuclear dacryocystogram. Journal of Nuclear Medicine, 16, 605-608.

Demorest, B. H., and Milder, B. (1955). Dacryocystography II. The pathologic lacrimal apparatus. Archives of Ophthalmology, 54, 410-421.

Ewing, A. E. (1909). Roentgen ray demonstrations of the lacrimal abscess cavity. American Journal of Ophthalmology, 26, 1-4.

Hurwitz, J. J., Maisey, M. N., and Welham, R. A. N. (1975a). Quantitative lacrimal scintillography II. Lacrimal pathology. British Journal of Ophthalmology, 59, 313-321.

Hurwitz, J. J., Maisey, M. N., and Welham, R. A. N. (1975b). Quantitative lacrimal scintillography I. Method and physiological application. British Journal of Ophthalmology, 59, 308-312.

Lloyd, G. A. S. (1973). Subtraction macryodacryocystography. Transactions of the Ophthalmological Societies of the United Kingdom, 93, 589-591.

Merriam, G. R., and Focht, E. F. (1957). A clinical study of radiation cataracts and the relationship to dose. American Journal of Roentgenology, 77, 759-785.

Rossomondo, R. M., Carlton, W. H., Trueblood, J. H., and Thomas, R. P. (1972). A new method of evaluating lacrimal drainage. Archives of Ophthalmology, 88, 523-525. 